\title{
Die einfache und komplexe Posttraumatische Belastungsstörung in der Praxis
}

\section{Eine Übersicht und Einordnung der neuen ICD-11 Kriterien in Bezug auf Kinder und Jugendliche}

\author{
Rebekka Eilers und Rita Rosner
}

Katholische Universität Eichstätt-Ingolstadt

\begin{abstract}
Zusammenfassung: Theoretischer Hintergrund: Die ICD-11 enthält reformulierte Kriterien für die Posttraumatische Belastungsstörung (PTBS) und die neue Diagnose komplexe PTBS (kPTBS). Fragestellung: Wie wirken sich die Neuerungen auf die Diagnostik und Behandlung von Kindern und Jugendlichen aus? Methode: In dieser Übersichtsarbeit werden die neuen Kriterien vorgestellt und mit früheren Diagnosemanualen verglichen. Bisherige Forschungsergebnisse zu PTBS ${ }_{I C D-11}$ und kPTBS bei Kindern und Jugendlichen werden zusammengefasst und diskutiert. Ergebnisse: Die PTBS $I_{I C D-11}$-Kriterien führen eher zu geringeren Prävalenzraten verglichen mit PTBS ${ }_{I C D-10}$, PTBS ${ }_{D S M-I V}$ und PTBS DSM-5. $_{1}$ Erste Studien weisen darauf hin, dass evidenzbasierte traumafokussierte Therapiemanuale auch zur Behandlung der kPTBS geeignet sind. Diskussion und Schlussfolgerung: Die Anwendung neuer Kriterien stellt Praktiker_innen und Forscher_innen vor Herausforderungen. Bisherige Ergebnisse deuten an, dass die kPTBS gut behandelbar ist.
\end{abstract}

Schlüsselwörter: Posttraumatische Belastungsstörung, komplexe Posttraumatische Belastungsstörung, diagnostische Kriterien, Traumatherapie, ICD-11

Simple and Complex Posttraumatic Stress Disorder in Clinical Practice: An Overview and Evaluation of the New ICD-11 Criteria for Children and Adolescents

Abstract: Theoretical Background: The $11^{\text {th }}$ version of the International Classification of Diseases (ICD-11) includes reformulated criteria for

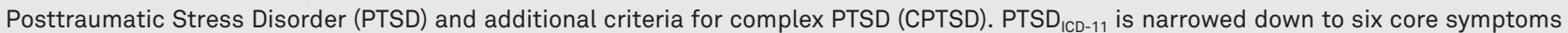
across three clusters; CPTSD is a distinct disorder comprising PTSD $_{I C D-11}$ criteria with additional criteria addressing disturbances in selforganization (DSO): affective dysregulation, negative self-concept, and interpersonal problems. Hence, therapists may be confronted in clinical practice with young patients with these new diagnoses, even though treatment recommendations are still not existent and randomized clinical trials have yet to be conducted. Objective: To give an overview for clinicians of PTSD ${ }_{\text {ICD-11 }}$ and CPTSD criteria for children and adolescents. Method: First, we present the diagnostic criteria for PTSD ${ }_{I C D-11}$ and CPTSD and discuss the diagnostic approach of PTSD ${ }_{I C D}$ 11 compared to PTSD $_{\text {DSM-IV }}$, PTSD $D_{D S M-5}$, and PTSD ${ }_{I C D-10}$. Second, we outline current research on PTSD $_{I C D-11}$ and $C P T S D$ in children and adolescents. We summarize the results on prevalence rates, on group differences between young patients with PTSD ICD-11 $_{\text {and }}$ CPTSD, and on the applicability of existing trauma-focused therapies for CPTSD. Third, we discuss possible implications for clinical practice and upcoming issues. Results: The PTSD ${ }_{\text {ICD-11 }}$ and CPTSD criteria are intended for use in all age groups, though developmentally adapted aspects are missing. PTSD $D_{I C D-11}$ incidence rates appear to be lower compared to PTSD $D_{D S M-I V}, P_{T S D_{D S M-5}}$, and PTSD $D_{I C D-10}$. Rates of PTSD $D_{I C D-11}$ and $C P T S D$ vary across populations; CPTSD rates appear to be lower than PTSD $_{1 C D-11}$ rates. Findings on group differences are heterogeneous. Studies indicate that trauma-focused cognitive behavioral therapy (TF-CBT) and developmentally adapted cognitive processing therapy (D-CPT) have a positive impact on PTSD ${ }_{I C D-11}$ and CPTSD symptoms. Discussion and conclusion: Results indicate that PTSD ${ }_{I C D-11}$ rates are stricter than diagnoses based on other versions of the PTSD criteria. Promising first results indicate that CPTSD symptoms can be treated successfully with existing trauma-focused treatment manuals. However, the benefit of additional treatment phases to address DSO symptoms needs further study. So far, data on PTSD ${ }_{1 C D-11}$ and CPTSD in children and adolescents are limited. Because most of the studies used archival data to map ICD-11 symptoms, their results have to be interpreted cautiously. A first screening questionnaire for PTSD ICD-11 $_{\text {and }}$ CPTSD has already been published and validated for children and adolescents. Further research using clinical interviews for PTSD ${ }_{I C D-11}$ and $C P T S D$ is necessary to gain further insight into the implications of the new diagnoses.

Keywords: posttraumatic stress disorder, complex posttraumatic stress disorder, diagnostic criteria, trauma-focused therapy, ICD-11

Die Diagnosekriterien der Posttraumatischen Belastungsstörung (PTBS) werden sich mit der ICD-11 (WHO,
2018, online abrufbar über https://icd.who.int/browse11/ $1-\mathrm{m} /$ en) deutlich verändern. Die PTBS ${ }_{\text {ICD-11 }}$-Kriterien wer- 


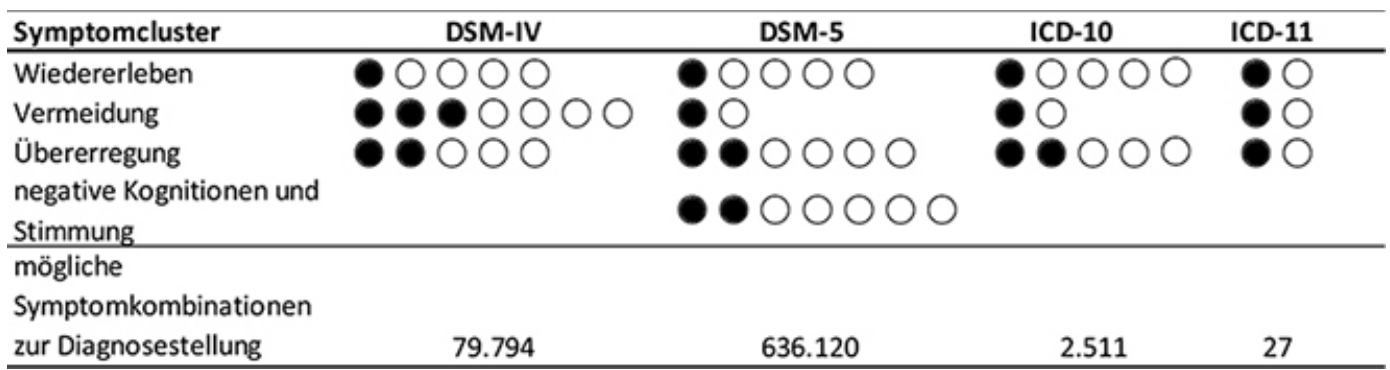

Anmerkung: Das Übererregungs-Cluster in den Forschungskriterien des ICD-10 ist erfüllt beim Vorliegen von entweder (1) Unfähigkeit, sich an wichtige Aspekte des traumatischen Ereignisses zu erinnern oder (2) zwei aus fünf weiteren Symptomen.

Abbildung 1. Übersicht zum jeweiligen diagnostischen Algorithmus der PTBS in den Diagnosemanualen mit der Anzahl beschriebener Symptome (leere Kreise), für eine Diagnosestellung notwendige Symptomzahl (ausgefültte Kreise) sowie mögliche Symptomkombinationen bei Erfüllung der Diagnose.

den auf sechs Kernsymptome reduziert und die komplexe PTBS (kPTBS) wird als neue Diagnose eingeführt. 2022 sollen die ICD-11-Kriterien in Kraft treten und eine Anwendung ist auch im deutschen Gesundheitssystem in Planung (BfArM, n.d.).

Bereits im Kindes- und Jugendalter ist die PTBS eine häufige psychische Störung. Über die Hälfte aller Kinder und Jugendlichen erleben bis zum 18. Lebensjahr ein traumatisches Ereignis (McLaughlin et al. 2013) und durchschnittlich $15.9 \%$ der Betroffenen entwickeln eine PTBS (Alisic et at. 2014). Für die Behandlung von PTBS bei jungen Patient_innen werden hohe Effektstärken berichtet (Morina, Koersen \& Pollet, 2016). Fachleute in Forschung und Praxis sind durch die anstehenden Änderungen in der ICD-11 vor die Herausforderung gestellt, die Gültigkeit bisheriger Evidenz und Leitlinien für die neuen Kriterien zu überprüfen.

Dieser Beitrag soll Praktiker_innen einen Überblick zu Befunden bezüglich PTBS $\mathrm{ICD}-11_{11}$ und kPTBS bei Kindern und Jugendlichen geben. Neben einer Vorstellung der Diagnosekriterien werden in einer narrativen Literaturübersicht bisherige Befunde zu Häufigkeiten, Gruppenunterschieden und Behandlung von kPTBS vorgestellt..

\section{Kriterien der PTBS in ICD10, DSM-IV und DSM-5}

Die Anzahl und Zusammenstellung der Symptome, mit denen die Diagnose einer PTBS beschrieben wird, hat sich in den vergangenen Jahren mit den Neuauflagen der beiden Diagnosemanuale ICD und DSM verändert.

Abbildung 1 bietet eine Übersicht über die diagnostischen Algorithmen für PTBS in den jeweiligen Diagnosemanualen mit der Anzahl möglicher Symptome, den für eine Diagnosestellung notwendigen Symptomen und der daraus resultierenden Anzahl möglicher Symptomkombinationen bei Erfüllung der Diagnose.

Kasten 1. Traumabezug der Symptome als Voraussetzung der Diagnosestellung

Voraussetzung für die PTBS-Diagnose ist in allen Manualen der Traumabezug der Symptome. Dies bedeutet, dass diese nach dem Erleben eines traumatischen Ereignisses erstmalig aufgetreten oder stärker geworden sind.

\section{ICD-10}

Die momentan im deutschen Gesundheitssystem angewandte ICD-10 (WHO, 1992) beschreibt PTBS (F43.1) in den drei Clustern Wiedererleben, Vermeidung und Übererregung und umfasst 13 Symptome. Symptome des Wiedererlebens sind Intrusionen, Alpträume, Flashbacks sowie physiologische Erregung und emotionale Belastung bei Erinnerung an das traumatische Ereignis. Vermeidung umfasst das aktive Meiden innerer (Gedanken) und äußerer (Personen, Orte) Erinnerungsreize. Übererregung wird beschrieben durch die Unfähigkeit, wichtige Aspekte des traumatischen Ereignisses zu erinnern, Schlafstörungen, erhöhte Reizbarkeit und Wut, Konzentrationsschwierigkeiten, Hypervigilanz und erhöhte Schreckhaftigkeit. Dauerhafte Veränderung der Persönlichkeit, die aus wiederholter oder langanhaltender Traumatisierung resultieren, können in der Diagnose Anhaltende Persönlichkeitsstörung nach Extrembelastung (F 62.0) abgebildet werden. Diese findet jedoch in der Forschung kaum Anwendung (Maercker et al., 2013). 
Tabelle 1. Diagnostische Kriterien nach ICD-11 für die Posttraumatische Belastungsstörung (PTBS) und komplexe PTBS (kPTBS)

\begin{tabular}{|c|c|}
\hline Symptomcluster & Beschreibung, Symptome \\
\hline \multicolumn{2}{|l|}{ PTBS } \\
\hline traumatisches Ereignis & $\begin{array}{l}\text { sehr bedrohliches oder schreckliches Ereignis oder eine Serie von } \\
\text { Ereignissen } \\
\text { kPTBS: meist langanhaltende oder wiederholte Ereignisse, aus denen eine Flucht schwierig oder unmöglich ist (z. B. } \\
\text { Folter, Sklaverei, wiederholte sexuelle oder körperliche Gewalt) }\end{array}$ \\
\hline Wiedererleben & $\begin{array}{l}\text { (1) intrusive Erinnerungen, Flashbacks oder (2) Alpträume, meist in Verbindung mit emotionaler Belastung oder phy- } \\
\text { sischen Reaktionen }\end{array}$ \\
\hline Vermeidung & $\begin{array}{l}\text { Vermeidung von (1) Gedanken und Erinnerungen oder (2) Aktivitäten, Situationen und Personen, die an das Ereignis } \\
\text { erinnern }\end{array}$ \\
\hline Übererregung & (1) Wahrnehmung anhaltender Bedrohung oder (2) erhöhte Schreckhaftigkeit \\
\hline Beeinträchtigung & funktionale Beeinträchtigung in persönlichen, familiären, sozialen oder anderen Bereichen \\
\hline \multicolumn{2}{|c|}{ zusätzlich zur Diagnose der kPTBS } \\
\hline $\begin{array}{l}\text { Schwierigkeiten der } \\
\text { Emotionsregulation }\end{array}$ & (1) erhöhte Reizbarkeit und Wut oder (2) verminderte emotionale Schwingungsfähigkeit \\
\hline verändertes Selbstbild & $\begin{array}{l}\text { (1) anhaltendes negatives Selbstbild, das dazu führt, dass die Person sich als beschädigt oder wertlos wahrnimmt oder } \\
\text { (2) tiefgreifende und anhaltende Gefühle von Scham, Schuld und Versagen }\end{array}$ \\
\hline $\begin{array}{l}\text { interpersonelle Schwie- } \\
\text { rigkeiten }\end{array}$ & anhaltende Schwierigkeiten, (1) Beziehungen aufrechtzuerhalten oder (2) sich anderen nahe zu fühlen. \\
\hline
\end{tabular}

Anmerkung: Für die Diagnosestellung muss in jedem Cluster eines von zwei Symptomen erfüllt sein.

\section{DSM-IV}

Der Großteil der PTBS-Forschung, insbesondere die oft über Jahre gehenden Therapiestudien, beruht auf den Kriterien des DSM-IV (APA, 2000). Diese umfassen 17 Symptome in drei Clustern: Wiedererleben, Vermeidung und Übererregung.

Studien zeigten jedoch, dass insbesondere bei wiederholter Traumatisierung die klassischen Kriterien der PTBS nicht ausreichten, um alle klinischen Erscheinungsformen abzubilden. Daher wurde die Berücksichtigung von zusätzlichen Kriterien einer komplexen Form der PTBS vorgeschlagen (Herman, 1992; Roth, Newman, Pelcovitz, van der Kolk \& Mandel, 1997), die aber im DSM-IV noch nicht in den Kriterienkatalog aufgenommen wurden.

\section{DSM-5}

Bei der DSM-5 Entwicklung (APA, 2013) wurden den PTBS $_{\text {DSM-IV }}$-Kriterien drei weitere Symptome hinzugefügt, die sich in Studien als klinisch relevant für die Behandlung einer PTBS oder den Schweregrad und die Chronizität der Diagnose erwiesen hatten (Friedman, 2013). Auf Grundlage faktorenanalytischer Befunde wurde ein viertes Symptomcluster zu negativen Veränderung von Kognitionen und Stimmung eingeführt und die Symptome wurden neu gegliedert. Damit enthält das DSM-5 einen weit gefassten Ansatz der PTBS mit insgesamt 20 Symptomen in vier Clustern. Für Kinder bis zu einem Alter von sechs Jahren wurden außerdem entwicklungsangepasste Kriterien formuliert.

\section{PTBS und kPTBS in der ICD-11}

\section{Vorstellung der Diagnosekriterien}

Das zentrale Ziel der ICD-11 war die Erhöhung der klinischen Nützlichkeit der diagnostischen Kriterien aller Störungsbilder (Reed, 2010). Klinische Nützlichkeit umfasst die klare und einfache Kommunizierbarkeit von Diagnosen, geringe Überschneidung von Kriterien und damit niedrigere Komorbiditätsraten, einfache klinische und interkulturelle Anwendbarkeit sowie die Nützlichkeit für Behandlungsentscheidungen.

Die Überarbeitung der PTBS unter Berücksichtigung dieser Vorgaben resultierte in der Reduzierung der Kriterien auf sechs Kernsymptome. Zur Berücksichtigung der Studienlage zu weiteren posttraumatischen Symptomen wurden Kriterien einer kPTBS formuliert. Die PTBS ${ }_{\text {ICD-11 }}{ }^{-}$ und kPTBS-Kriterien sind in Tabelle 1 dargestellt.

Die PTBS ${ }_{\mathrm{ICD}-11}$ wird durch jeweils zwei Symptome in drei Clustern beschrieben. Mindestens ein Symptom aus jedem Bereich muss zur Diagnosestellung erfüllt sein. Die PTBS $_{\text {ICD-11 }}$ kann nach einzelnen oder multiplen potentiell traumatischen Ereignissen auftreten, die als sehr bedrohlich oder schrecklich empfunden werden. Zudem muss eine funktionale Beeinträchtigung in verschiedenen Le- 
bensbereichen vorliegen. Die sechs PTBS ICD-11-Symptome waren auch in früheren Versionen der Diagnosemanuale enthalten (PTBS ICD-10, $_{\text {PTBS }}$ DSM-IV und PTBS DSM-5 $_{\text {. }}$. Kriterien, die sich mit anderen Störungsbildern überschneiden, wurden gestrichen (z. B. Schlafprobleme, Konzentrationsschwierigkeiten; Maercker et al., 2013).

Die kPTBS Diagnose ist hierarchisch strukturiert. Sie wird beschrieben durch die Erfüllung der PTBS ${ }_{\text {ICD-11-Kri- }}$ terien und durch zusätzliche sogenannte Schwierigkeiten in der Selbstorganisation (SSO, disturbances in self-regulation) bezüglich Emotionsregulation, Selbstbild und interpersonellen Beziehungen. Jeder dieser Bereiche umfasst zwei Symptome, wovon jeweils mindestens eines für die Diagnosestellung vorliegen muss. Die SSO repräsentieren stressinduzierte und tiefgreifende Beeinträchtigungen, deren Auftreten nicht an traumaassoziierte Reize gebunden ist.

Kasten 2. Differentialdiagnostische Merkmale der PTBS $\left.\right|_{1 C D-11}$ und kPTBS

Treten Schwierigkeiten in der Selbstregulation ohne eine zusätzliche PTBS ${ }_{I C D-11}$ auf, dann liegt keine kPTBS vor.

Auch die kPTBS kann nach einzelnen potenziell traumatischen Ereignissen folgen, ist jedoch häufiger mit langanhaltenden oder wiederholten Ereignissen assoziiert (Maercker et al., 2013).

Die SSO-Symptome sind nur teilweise neu, da einzelne Kriterien bereits in früheren Versionen der PTBS-Diagnose enthalten waren. Reizbarkeit und Wut waren bei

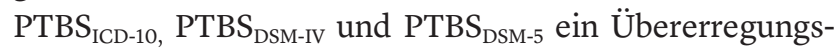
symptom. Verminderte Emotionalität, Schuld und Schwierigkeiten Beziehungen aufrechtzuerhalten, haben Ähnlichkeiten zu Symptomen aus dem PTBS DSM-5 -Cluster der negativen Veränderung von Kognitionen und Stimmung, wie die Unfähigkeit, positive Gefühle zu empfinden, verzerrte Kognitionen zu Ursachen und Folgen des traumatischen Ereignisses und das Gefühl der Entfremdung.

PTBS $_{\text {ICD-11 }}$ und kPTBS werden in einem neuen Kapitel disorders specifically associated with stress (mit Stress assoziierte Störungen) aufgeführt, in welchem auch die Anhaltende Trauerstörung, Anpassungsstörung, reaktive Bindungsstörung und Bindungsstörung mit Enthemmung enthalten sind (WHO, 2018).

\section{Vergleich der Diagnosemanuale}

Mit den Neuauflagen von DSM und ICD sollen aktuelle Forschungsbefunde berücksichtigt und Nachteile der bisherigen Klassifizierung ausgeglichen werden. Den Überarbeitungen wurden unterschiedliche Maßgaben zugrunde gelegt. Dies resultierte einerseits in einer Ausweitung der PTBS ${ }_{\text {DSM-5 }}$ auf 20 Kriterien, wobei die Diagnose nun die sogenannten komplexen Symptome besser erfasst, und andererseits in einem hierarchischen Ansatz in der ICD-11 mit wenigen PTBS-Symptomen und einer zusätzlichen kPTBS-Diagnose.

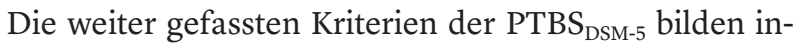
nerhalb einer Diagnose vielfältige klinische Erscheinungsformen ab, wodurch jedoch die Patient_innengruppe mit dieser Störung heterogener wird. Ein reduzierter Kriterienkatalog wie die PTBS ${ }_{\text {ICD-11 }}$ erfasst weniger Ausprägungen, ist dadurch aber ökonomischer in der Anwendung und die reduzierte Überschneidung mit Symptomen anderer Störungen kann die differenzialdiagnostische Abgrenzung erleichtern. Die größer gewordenen Unterschiede stellen die Kommunikation von Forscher_innen, welche vor allem das DSM-5 anwenden, und Praktiker_innen, die mit der ICD-11 arbeiten werden, vor neue Herausforderungen.

\section{Entwicklungsbezogene Aspekte}

Bereits bei vorherigen PTBS-Versionen wurde kritisiert, dass diese im Kindesalter zu einer bedeutenden Unterschätzung der Diagnose führen, während auch bei subklinischer Symptomatik eine hohe funktionale Beeinträchtigung vorliegt (Übersicht bei Steil \& Rosner, 2009). Dies kann auch sozialrechtliche Auswirkungen haben. Daher wird empfohlen, bei Kindern nicht die vollständigen Kriterien anzuwenden. Die PTBS DSM-5 $_{\text {berücksichtigt }}$ entwicklungsangepasste Kriterien lediglich für Vorschulkinder. In der Version der PTBS ${ }_{\mathrm{ICD}-11}$ sind keine entwicklungsangepassten Symptome aufgeführt.

Als Abweichung von den oben beschriebenen Kriterien sind im Kindesalter Albträume ohne klaren Inhalt, Trennungsängste und der Verlust bereits erworbener Fähigkeiten zu erwarten. Vorschulkinder zeigen eingeengtes, repetitives Spiel und sozialen Rückzug. Bei Jugendlichen treten Probleme der Emotionsregulation, Substanzmissbrauch sowie selbstverletzendes und riskantes Verhalten auf (Jensen, Cohen, Jaycox \& Rosner, 2020). 


\section{Differenzialdiagnostische Abgrenzung der kPTBS}

Mit der Einführung der kPTBS muss auch die differenzialdiagnostische Abgrenzung der neuen SSO-Kriterien diskutiert werden. Bei Erwachsenen ist die Abgrenzung zur Borderline-Persönlichkeitsstörung schwierig (Jowett, Karatzias, Shevlin \& Albert, 2020) und kann auch bei Jugendlichen herausfordernd sein. Im Kindes- und Jugendalter stellt sich die Frage der Abgrenzung von pathologischen SSO-Symptomen und entwicklungsbedingten Schwierigkeiten bei Emotionsregulation, Selbstbild und Beziehungsgestaltung (Jensen et al., 2020). Da die SSOSymptome nicht traumaspezifisch sind, muss außerdem beachtet werden, ob sie mit dem traumatischen Ereignis auftraten, bereits vorher als Risikofaktor vorlagen oder einer anderen Störung zugeordnet sind.

\section{Forschungsergebnisse zu PTBS ICD-11 $_{11}$ und kPTBS bei Kindern und Jugendlichen}

Die Studienlage zu PTBS ICD-11 und kPTBS bei Kindern und Jugendlichen wächst seit der ersten Vorstellung der Kriterien durch Maercker et al. (2013) stetig an. Ziel dieses Abschnittes ist es, eine Übersicht der bisherigen Forschungslage zu folgenden Fragestellungen zu geben: Wie häufig erfüllen Kinder und Jugendliche die PTBS ${ }_{\text {ICD-11 }}$ im Vergleich zu PTBS ${ }_{\text {ICD-10 }}$, PTBS ${ }_{\text {DSM-IV }}$ und PTBS DSM-5 $_{\text {? Wie }}$ viele Kinder und Jugendliche erfüllen die Kriterien der kPTBS? In welchen Merkmalen unterscheiden sich Pati-

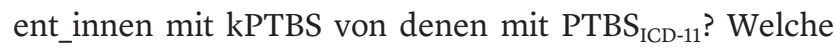
Behandlungsmöglichkeiten gibt es für Kinder und Jugendliche mit kPTBS?

\section{Häufigkeit der PTBS ICD-11 $_{1 m}$ Vergleich zu anderen Versionen der PTBS-Kriterien}

Die Veränderung von Störungskriterien und diagnostischen Algorithmen kann sich zunächst auf die Häufigkeit einer Diagnose auswirken. Um diesen Aspekt zu untersuchen, wurden die jeweiligen Kriterien einer PTBS ICD-11, $_{\text {, }}$ PTBS $_{\text {ICD-10 }}$, PTBS DSM-IV $_{\text {und }}$ PTBS ${ }_{\text {DSM-5 }}$ bei verschiedenen Stichproben angelegt.

Eine Sekundäranalyse berichtet deutlich geringere Raten (Differenzen 10-35\%) für PTBS ${ }_{\text {ICD-11 }}$ verglichen mit PTBS $_{\text {DSM-IV }}$ und PTBS ${ }_{\text {ICD-10 }}$ in einer klinischen Stichprobe mit unterschiedlichen traumatischen Ereignissen (717 Jahre; Sachser \& Goldbeck, 2016). PTBS ${ }_{\text {ICD-11 }}$ hatte auch eine geringere Häufigkeit verglichen mit PTBS $_{\mathrm{DSM}-\mathrm{IV}}$, PTBS $_{\text {DSM-5 }}$ und PTBS ICD-10 $_{10}$ bei jungen Patient_innen nach sexualisierter und physischer Gewalt (14-21 Jahre; Eilers, Rimane, Vogel, Renneberg, Steil \& Rosner, 2020). In weiteren Studien mit klinischen Stichproben waren die Ergebnisse heterogener. Eine Sekundäranalyse bei Patient_innen (3-6 Jahre) nach unterschiedlichen traumatischen Ereignissen in Deutschland und der Schweiz zeigte

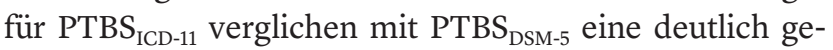
ringere Rate in der deutschen, nicht jedoch in der schweizerischen Gruppe (Vasileva, Haag, Landolt \& Petermann, 2018). Eine längsschnittliche Erhebung mit Kindern und Jugendlichen (8-17 Jahre) nach einmaligen traumatischen Ereignissen, die einen für PTBS $_{\text {ICD-11 }}$ und kPTBS validierten Fragebogen einsetzte, berichtete zwei Wochen nach dem Ereignis mehr als doppelt so hohe Häufigkeiten für PTBS ICD-10 $_{\text {PTBS }}$ DSM-IV und PTBS DSM-5 $_{\text {D }}$ verglichen mit PTBS ${ }_{\text {ICD-11 }}$. Neun Wochen nach dem Ereignis waren die Häufigkeiten gemäß aller PTBS-Versionen deutlich gesunken und die Unterschiede waren nur noch gering. Die geringste Häufigkeit hatte weiterhin PTBS $_{\text {ICD-11 }}$ (Elliott et al. 2020).

In zwei Sekundäranalysen nicht-klinischer Stichproben wurden annähernd gleiche Häufigkeiten (Differenzen unter $3 \%$ ) berichtet. Eine Studie bei Jugendlichen und jungen Erwachsenen (13 Jahre und älter) verglich PTBS $_{\text {ICD-11 }}$ und PTBS ${ }_{\text {DSM-5 }}$ nach einem Terroranschlag (Hafstad, Thoresen, Wetzel-Larsen, Maercker \& Dyb, 2017). Eine weitere Studie verglich PTBS $_{\text {DSM-IV }}$, PTBS $_{\text {DSM-5 }}$ und PTBS ${ }_{\text {ICD-11 }}$ bei Kindern zwischen 7 und 11 Jahren nach Hurrikanen (Danzi \& La Greca, 2016).

Trotz ähnlicher Häufigkeitsraten war die diagnostische Übereinstimmung der verschiedenen PTBS-Kriterien bei Danzi und La Greca (2016) gering. Nur ein Drittel aller Teilnehmenden erfüllte zugleich PTBS ICD-11 $_{1}$ PTBS $_{\text {ICD-10, }}$,

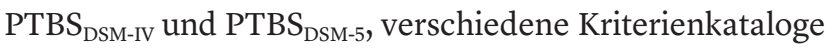
erfassten unterschiedliche Patient_innen. Insgesamt sind die Befunde zur diagnostischen Übereinstimmung heterogen und reichen von geringer (Eilers et al., 2020; Danzi \& La Greca, 2016) bis guter (Hafstad et al., 2017) Übereinstimmung.

\section{Häufigkeit von PTBS ${ }_{I C D-11}$ und kPTBS}

Tabelle 2 stellt bisherige vergleichende Forschungsergebnisse zur Häufigkeit von PTBS ICD-11 $_{11}$ und kPTBS bei Kindern und Jugendlichen dar.

Komplexe PTBS wurde in verschiedenen Stichproben untersucht: Sowohl bei Kindern und Jugendlichen nach einzelnen traumatischen Ereignissen (Elliott et al., 2020), als auch nach sexuellen und physischen Gewalterfahrungen (Eilers et al., 2020; Hébert \& Amédée, 2020), bzw. nach 
Tabelle 2. Prävalenzraten von PTBS nach ICD-11 und kPTBS bei Kindern und Jugendlichen

\begin{tabular}{|c|c|c|c|c|}
\hline \multirow[b]{2}{*}{ unterschiedliche traumatische Ereignisse } & \multirow{2}{*}{$\begin{array}{l}\text { Autoren } \\
\text { Elliott et al., } 2020\end{array}$} & \multirow{2}{*}{$\begin{array}{l}\text { Stichprobe } \\
n=262,8-14(M=14.1) \text { Jahre }\end{array}$} & \multicolumn{2}{|c|}{ Prävalenzraten } \\
\hline & & & PTBS & $6.6 \%$ \\
\hline & & & kPTBS & $4.8 \%$ \\
\hline & Kazlauskas et al., 2020 & $n=932,12-16(M=14.25)$ Jahre & PTBS & $12.6 \%$ \\
\hline & & & kPTBS & $32.4 \%$ \\
\hline & Sachser et al., 2017 & $n=159,7-17(M=13.01)$ Jahre & PTBS* & $59.4 \%$ \\
\hline & & & kPTBS & $40.6 \%$ \\
\hline & Villalta et al., 2020 & $n=134$ Mädchen, $13-17(M=14.25)$ Jahre & PTBS & $59 \%$ \\
\hline & & & kPTBS & $40 \%$ \\
\hline \multirow[t]{8}{*}{ interpersonelle traumatische Ereignisse } & Eilers et al., 2020 & $n=83,14-21(M=17.65)$ Jahre & PTBS & $16.87 \%$ \\
\hline & & & kPTBS & $49.40 \%$ \\
\hline & Haselgruber et al., 2020 & $n=139,10-18(M=14.28)$ Jahre & PTBS & $31.6 \%$ \\
\hline & & & kPTBS & $22.8 \%$ \\
\hline & Hébert \& Amédée, 2020 & $n=384,6-14(M=9.56)$ Jahre & PTBS & $51.3 \%$ \\
\hline & & & kPTBS & $23.4 \%$ \\
\hline & Perkonigg et al., 2016 & $n=640,14-24$ Jahre & PTBS & $19.4 \%$ \\
\hline & & & kPTBS & $8.2 \%$ \\
\hline
\end{tabular}

Anmerkung: *Die PTBS-Diagnose in dieser Studie entspricht nicht den ICD-11-Kriterien, sondern einem Summenwert $\geq 35$ im Interview zu Belastungsstörungen bei Kindern und Jugendlichen (IBS-KJ).

Gewalt und Vernachlässigung (Haselgruber, Sölva \& Lueger-Schuster., 2020) sowie in gemischten Stichproben (Kazlauskas et al., 2020; Sachser, Keller \& Goldbeck, 2017; Villalta et al, 2020). Die meisten Studien berichte-

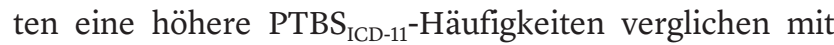
kPTBS (Elliott et al., 2020; Haselgruber et al., 2020; Hébert \& Amédée, 2020; Perkonigg, Höfler, Cloitre, Wittchen, Trautmann \& Maercker, 2016; Sachser et al., 2017; Villalta et al., 2020), wobei die Differenzen der Inzidenzraten variierten. Zwei weitere Studien berichteten höhere Häufigkeitsraten für kPTBS (Eilers et al., 2020; Kazlauskas et al., 2020). In allen Stichproben wurden auch ein-

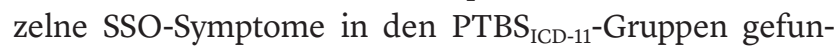
den, auch wenn diese nicht die vollen kPTBS-Kriterien erfüllten.

\section{Unterschiede zwischen Kindern und Jugendlichen mit PTBS ${ }_{\mathrm{ICD}-11}$ und kPTBS}

Neben dem Erfüllen der zusätzlichen SSO-Symptome unterscheiden sich Patient_innen mit kPTBS von denen mit PTBS $_{\text {ICD-11 }}$ durch mehr komorbide Diagnosen (Eilers et al., 2020; Perkonigg et al., 2016), wie Depression (Haselgruber et al., 2020; Sachser et al., 2017), Generalisierter Angststörung (Haselgruber et al., 2020) und Dissoziation (Eilers et al., 2020). Die Diagnose einer kPTBS trat häufiger bei Mädchen auf (Hébert \& Amédée, 2020; Sachser et al., 2017) sowie nach interpersoneller Gewalt (Sachser et al., 2017) und einer größeren Anzahl traumatischer Er- eignisse (Haselgruber et al., 2020). Komplexe PTBS hing mit größerer Beeinträchtigung (Haselgruber et al., 2020; Perkonigg et al., 2016), mehr Verhaltensproblemen und maladaptiver Emotionsregulation (Haselgruber et al., 2020) zusammen.

\section{Behandlung der kPTBS}

Mit Einführung der kPTBS stehen jedoch spezifische Behandlungsempfehlungen für diese Störung noch aus. Zudem muss die Nützlichkeit einer neuen Diagnose auch daran gemessen werden, wie hilfreich sie zur Therapieplanung ist. Bei der kPTBS stellt sich angesichts der teilweise mit PTBS $\mathrm{ICD}-11_{11}$ übereinstimmenden Kriterien die Frage, ob evidenzbasierte traumafokussierte Psychotherapien zur Behandlung der kPTBS ausreichen oder ob zusätzliche oder andere Interventionen notwendig sind.

Ein Therapieprogramm, welches zur Behandlung von kPTBS bei Erwachsenen empfohlen wird, kombiniert Skills-Training zur affektiven und interpersonellen Regulation mit Narrativer Therapie (STAIR-NT Cloitre, Cohen \& Koenen, 2006; Schäfer, Borowski \& Cloitre, 2019a). In einer ersten Behandlungsphase werden SSO adressiert und in einer zweiten Phase folgen ein Traumanarrativ und Skills-Training mit Bezug auf Alltagsprobleme. Studien zur Effektivität von STAIR-NT für kPTBS stehen noch aus.

Aufgrund unzureichender Datenlage existieren bislang noch keine Leitlinien oder andere Behandlungsempfeh- 
lungen für die kPTBS bei Kindern und Jugendlichen (Jensen et al., 2020 Schäfer et al., 2019b). Zur Behandlung von PTBS bei Kindern und Jugendlichen liegt für die traumafokussierte kognitive Verhaltenstherapie und insbesondere für das Behandlungsmodell nach Cohen, Mannarino und Deblinger, 2009 (TF-KVT) die meiste Evidenz vor (Morina et al., 2016). TF-KVT wird in den deutschen (Schäfer et al., 2019b) und internationalen Leitlinien (NICE, 2018) empfohlen (für eine aktuelle Übersicht Steil, Fischer \& Rosner, 2021). Die TF-KVT besteht aus acht Komponenten: Psychoedukation, Entspannung, Ausdruck und Modulation von Effekten, Kognitive Verarbeitung und Bewältigung, Traumanarrativ, in-vivo Konfrontation, gemeinsame Eltern-Kind-Sitzungen und die Verbesserung künftiger Sicherheit. Das Manual sieht Einzelsitzungen mit dem Kind und einer Bezugsperson wie auch gemeinsame Sitzungen vor.

Als erste Annäherung an Therapiemöglichkeiten für kPTBS bei Kindern und Jugendlichen wurde in zwei Studien die Anwendbarkeit der TF-KVT untersucht. Sachser et al. (2017) untersuchten 61 Teilnehmende (7-17 Jahre), welche die Therapie im Rahmen einer randomisiert-kontrollierten Studie (RCT) abgeschlossen hatten. Diese wurden post-hoc einer kPTBS- oder PTBS DSM-IV $_{\text {-Gruppe }}$ zugeordnet. Die posttraumatische Stresssymptomatik (PTSS) verbesserte sich mit großer Effektstärke (PTBS: $d$ $=2.81$, kPTBS: $d=1.37$ ), wobei Teilnehmende in der kPTBS-Gruppe vor und nach der Therapie eine höhere PTSS berichteten. SSO-Symptome der kPTBS-Gruppe verbesserten sich mit kleiner bis großer Effektstärke $(d=$ $0.40-1.16)$.

Hébert und Amédée (2020) untersuchten Verläufe von 326 Kindern im Alter zwischen 6 und 14 Jahren, die nach physischer oder sexueller Gewalt und Vernachlässigung mit TF-KVT behandelt wurden. Diese wurden drei Gruppen zugeteilt: PTBS $_{\text {ICD-11 }}$, kPTBS und resilient. In der kPTBS-Gruppe verbesserte sich die PTSS mit großer Effektstärke $(d=0.90)$, Dissoziationssymptome, internalisierende und externalisierende Verhaltensprobleme mit mittleren Effektstärken $(d=0.76-0.78)$. In der PTBSGruppe verbesserten sich alle untersuchten Symptombereiche mit mittlerer Effektstärke $(d=0.50-0.66)$. In der resilienten Gruppe veränderte sich die ohnehin geringe PTSS nicht $(d=0.02)$ und komorbide Symptome verbesserten sich mit mittlerer Effektstärke $(d=0.57-0.78)$.

Bei Jugendlichen und jungen Erwachsenen wurde in einer Sekundäranalyse die entwicklungsangepasste Kognitive Verhaltenstherapie (E-KVT; Matulis, Resick, Rosner \& Steil, 2014) für kPTBS untersucht (Eilers, Rimane, Vogel, Renneberg, Steil \& Rosner, 2021). Die E-KVT adressiert neben der PTSS in weiteren Phasen auch SSOSymptombereiche (Emotionsregulation, Beziehungsgestaltung). Die Behandlungsgruppe $(N=44,14-21$ Jahre $)$ eines RCTs wurde in eine kPTBS- und eine PTBS $_{\text {DSM-IV }^{-}}$ Gruppe geteilt. Die Auswertung der Symptomverläufe zeigte eine Verbesserung der PTSS in beiden Gruppen (kPTBS: $d=1.39$, PTBS $_{\text {DSM-Iv }}: d=1.80$ ). In allen drei hier beschriebenen Studien hatte die kPTBS-Gruppe vor und nach der Therapie höhere PTSS-Werte als die PTBSGruppe.

\section{Diskussion der dargestellten Forschungsergebnisse}

Die bisherigen Befunde geben einen ersten Einblick, welche Implikationen die neuen Kriterien der PTBS ICD-11 $_{\text {und }}$ kPTBS haben werden. Beide Störungsbilder können nach einmaligen oder mehrmaligen traumatischen Ereignissen auftreten. Die Häufigkeitsraten unterschiedlicher PTBSVersionen unterscheiden sich je nach Stichprobe, sind jedoch bei PTBS ICD-11 $_{\text {im Vergleich zu PTBS }}$ ICD-10, PTBS $_{\text {DSM-IV }}$ und PTBS ${ }_{\text {DSM-5 }}$ meist geringer. Dies wird auch bei Erwachsenen berichtet (Brewin et al., 2017). Mit Anwendung der PTBS ${ }_{\text {ICD-11 }}$ könnten junge Patient_innen mit klinisch relevanter PTSS eine Diagnose verfehlen und damit keine Indikation für eine traumafokussierte Therapie erhalten.

Erste Ergebnisse deuten darauf hin, dass kPTBS bei Kindern und Jugendlichen mit TF-KVT und E-KVT behandelt werden kann. Die Manuale erreichen große Effektstärken und es bilden sich auch komorbide Symptome zurück. Allerdings starteten und beendeten Patient_innen mit kPTBS die Therapie offensichtlich auf höherem posttraumatischen Symptomniveau. Offen bleibt die Frage, ob junge Patient_innen mit kPTBS von zusätzlichen Interventionen profitieren würden. Bei Erwachsenen gibt es erste Hinweise hierfür, jedoch untersuchte der Großteil der Studien nur einzelne SSO-Symptomcluster und nicht die gesamte kPTBS (Schäfer et al., 2019b).

Die vorliegende Literatursuche erfolgte nicht systematisch und bietet nur einen ersten narrativen Überblick. Eine zentrale Limitation ist die Art der Diagnosestellung in den meisten Studien. Da noch keine validierten klinischen Interviews und Fragebögen vorlagen, wurden für sekundäranalytisch ausgewertete PTBS ${\mathrm{ICD}-11^{-}}^{-}$und kPTBSDiagnosen Items anderer Fragebögen genutzt. So können die berichteten Daten nur als Annäherungswerte interpretiert werden. Die kPTBS-Häufigkeiten wurden nur in den Studien berechnet, die in Tabelle 2 dargestellt sind. Für eine differenzierte Betrachtung der Ergebnisse für verschiedene Altersgruppen im Kindes- und Jugendalter ist die Studienlage noch zu gering.

Weitere Forschung wird notwendig sein, um bisherige Ergebnisse zu bestätigen, Häufigkeiten genauer abzubilden und Grundlagen für die Behandlung von jungen Pa- 
tient_innen mit kPTBS zu schaffen. Dabei müssen PTBS $_{\text {ICD-11 }}$ und kPTBS mit klinischen Interviews diagnostiziert werden. Auch Befunde dazu, ob Patient_innen, die eine PTBS ${ }_{\text {ICD-11 }}$ verfehlen, eine andere Diagnose und Behandlungsindikation erhalten werden, stehen noch aus.

\section{Zusammenfassung: Welche Implikationen haben die Neuerungen für die klinische Praxis?}

\section{Patient_innen mit PTBS $\mathrm{ICD}-11_{11}$ und kPTBS}

Die PTBS ${ }_{\text {ICD-11 }}$ wird auf sechs Kriterien reduziert. Verglichen mit PTBS ${ }_{\text {ICD-10 }}$, PTBS ${ }_{\text {DSM-IV }}$ und PTBS DSM-5 $_{\text {werden die }}$ Prävalenzraten sinken.

Als neue Diagnose wird zudem die kPTBS eingeführt, sie wird durch die PTBS ${ }_{\text {ICD-11 }}$-Kriterien und zusätzliche SSO beschrieben. Ein Teil der bisherigen PTBS-Patient_innen wird zukünftig die Diagnose kPTBS erhalten. Bisherige Befunde $\mathrm{zu}$ den Unterschieden zwischen PTBS $_{\text {ICD-11 }}$ und kPTBS sind heterogen und es ist unklar, ob die kPTBS eine eigene Patient_inenngruppe erfasst oder einen höheren Schweregrad der PTBS.

\section{Diagnostik}

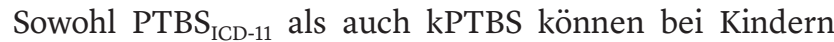
und Jugendlichen nach einmaligen oder mehrmaligen traumatischen Ereignissen auftreten. Die aktuell publizierten Diagnosekriterien gelten ohne Altersbeschränkung (verfügbar über www.icd.who.int/browse11/l-m/ en). Die Reduktion auf wenige Kriterien erleichtert eine schnelle Diagnosestellung.

Für eine angemessene Diagnostik fehlen noch entwicklungsangepasste Kriterien. Gerade bei Kindern und Jugendlichen ist zudem eine Abgrenzung der SSO-Symptome von entwicklungsbedingten Schwankungen der Emotionsregulation, des Selbstbilds und der Beziehungsgestaltung erforderlich. Zum Screening für PTBS ${ }_{\text {ICD-11 }}$ und kPTBS bei Kindern und Jugendlichen steht in deutscher Sprache der International Trauma Questionnaire (ITQ Cloitre et al., 2018; Haselgruber et al., 2020) zur Verfügung (unter www.traumameasuresglobal.com/itq). Dieser Selbstauskunfts-Fragebogen erfasst die Symptombelastung im letzten Monat für die zwölf Symptome der PTBS $_{\text {ICD-11 }}$ und kPTBS. Ein weiteres Screeningmaß mit Selbst- und Fremdbeurteilung (Child and Adolescent Trauma Screen-2, CATS-2) befindet sich derzeit in der Evaluationsphase und wird unter www.ulmer-onlinekli nik.de verfügbar sein. Die Entwicklung und Validierung eines klinisches Interview für PTBS ICD-11 $_{11}$ wird aktuell durchgeführt (Lechner-Meichsner \& Steil, 2021). Für Kinder und Jugendliche liegt in deutscher Sprache das Goldstandardinstrument IBS-KJ bisher nur für PTBS ${ }_{\text {DSM-5 }}$ und PTBS ${ }_{\text {ICD-10 }}$ vor (Steil \& Füchsle, 2006).

\section{Behandlung von kPTBS}

Bisherige Befunde deuten darauf hin, dass auch die kPTBS mit TF-KVT und E-KVT behandelt werden kann. Diese führten zu einer Reduktion sowohl der PTBS ICD-11- $^{-}$ als auch der kPTBS-Symptomatik.

\section{Schlussfolgerung}

In der Praxis lassen sich junge Patient_innen mit bekannten traumafokussierten Manualen behandeln. Weitere Forschung ist unerlässlich, um die ersten Befunde $\mathrm{zu}$ PTBS $_{\text {ICD-11 }}$ und kPTBS zu validieren sowie noch offene Fragen zu klären. Wichtig sind hierbei vor allem die Entwicklung eines angepassten klinischen Interviews zur gesicherten Diagnosestellung, die Überprüfung der Altersangemessenheit der Kriterien und die Nützlichkeit der kPTBS als distinkte Diagnose. Adaptionen bestehender bzw. die Neuentwicklung von Therapien sollten empirisch abgesichert werden.

\section{Literatur}

Alisic, E., Zalta, A. K., van Wesel, F., Larsen, S. E., Hafstad, G. S., Hassanpour, K., et al. (2014). Rates of posttraumatic stress disorder in trauma-exposed children and adolescents: metaanalysis. The British Journal of Psychiatry, 204, 335-340. https://doi.org/10.1192/bjp.bp.113.131227

American Psychiatric Association (APA). (2000). Diagnostic and statistical manual of mental disorders, DSM-IV-TR. Washington, DC: American Psychiatric Association.

American Psychiatric Association (APA). (2013). Diagnostic and statistical manual of mental disorders (5th ed.). Washington, DC: American Psychiatric Association.

Brewin, C. R., Cloitre, M., Hyland, P., Shevlin, M., Maercker, A., Bryant, R. A. et al. (2017). A review of current evidence regarding the ICD-11 proposals for diagnosing PTSD and complex PTSD. Clinical Psychology Review, 58, 1 -15. https://doi.org/10.1016/j. cpr.2017.09.001

Bundesinstitut für Arzneimittel und Medizinprodukte (BfArM). (n. d.). Wann kommt die ICD-11? Verfügbar unter https://www. dimdi.de/dynamic/de/faq/faq/Wann-kommt-die-ICD-11/

Cloitre, M., Cohen, L. R. \& Koenen, K. C. (2006). Treating survivors of childhood abuse. London, UK: Guilford Press. 
Cloitre, M., Shevlin, M., Brewin, C. R., Bisson, J. I., Roberts, N. P., Maercker, A. et al. (2018). The International Trauma Questionnaire. Development of a self-report measure of ICD-11 PTSD and complex PTSD. Acta Psychiatrica Scandinavica, 138, 536 - 546. https://doi.org/10.1111/acps.12956

Cohen, J. A., Mannarino, A. P. \& Deblinger, E. (2009). Traumafokussierte kognitive Verhaltenstherapie bei Kindern und Jugendlichen. Heidelberg: Springer.

Danzi, B. A. \& La Greca, A. M. (2016). DSM-IV, DSM-5, and ICD-11. Identifying children with posttraumatic stress disorder after disasters. Journal of Child Psychology and Psychiatry, and Allied Disciplines, 57, 1444-1452. https://doi.org/10.1111/jcpp.12631

Eilers, R., Rimane, E., Vogel, A., Renneberg, B., Steil, R. \& Rosner, R. (2020). The impact of the new ICD-11 criteria on abused young people. $30 \%$ less PTSD and CPTSD diagnoses compared to DSM-IV. Psychotherapy and Psychosomatics, 89, 59-61. https://doi.org/10.1159/000503794

Eilers, R., Rimane, E., Vogel, A., Renneberg, B., Steil, R. \& Rosner, R. (2021). Response of young patients with probable ICD-11 Complex PTSD to treatment with developmentally adapted cognitive processing therapy. European Journal of Psychotraumatology, 12(1). https://doi.org/10.1080/20008198.2021.1929024

Elliott, R., McKinnon, A., Dixon, C., Boyle, A., Murphy, F., Dahm, T. et al. (2020). Prevalence and predictive value of ICD-11 posttraumatic stress disorder and complex PTSD diagnoses in children and adolescents exposed to a single-event trauma. Journal of Child Psychology and Psychiatry and Allied Disciplines, 41, 166 - 173. https://doi.org/10.1111/jcpp.13240

Friedman, M. J. (2013). Finalizing PTSD in DSM-5: Getting here from there and where to go next. Journal of Traumatic Stress, 26, 548 -556. https://doi.org/10.1002/jts.21840

Hafstad, G. S., Thoresen, S., Wentzel-Larsen, T., Maercker, A. \& Dyb, G. (2017). PTSD or not PTSD? Comparing the proposed ICD11 and the DSM-5 PTSD criteria among young survivors of the 2011 Norway attacks and their parents. Psychological Medicine, 47, 1283 - 1291. https://doi.org/10.1017/S0033291716002968

Haselgruber, A., Sölva, K. \& Lueger-Schuster, B. (2020). Validation of ICD-11 PTSD and complex PTSD in foster children using the International Trauma Questionnaire. Acta Psychiatrica Scandinavica, 141, 60 - 73. https://doi.org/10.1111/acps.13100

Hébert, M. \& Amédée, L. M. (2020). Latent class analysis of posttraumatic stress symptoms and complex PTSD in child victims of sexual abuse and their response to trauma-focused cognitive behavioural therapy. European Journal of Psychotraumatology, 11, 1807171. https://doi.org/10.1080/20008198.2020.1807171

Herman, J. L. (1992). Complex PTSD: A syndrome in survivors of prolonged and repeated trauma. Journal of Traumatic Stress, 5, 377 - 391. https://doi.org/10.1002/jts.2490050305

Jensen, T., Cohen, J., Jaycox, L. \& Rosner, R. (2020). Treatments for children and adolescents. In D. Forbes, J. I. Bisson, C. M. Monson \& L. Berliner (Eds.), Effective Treatments for PTSD: practice guidelines from the International Society of Traumatic Stress Studies (3rd ed., pp. 385-416). New York: Guilford.

Jowett, S., Karatzias, T., Shevlin, M. \& Albert, I. (2020). Differentiating symptom profiles of ICD-11 PTSD, complex PTSD, and borderline personality disorder: A latent class analysis in a multiply traumatized sample. Personality Disorders: Theory, Research, and Treatment, 11 (1), 36 -45. https://doi.org/10. 1037/per0000346

Kazlauskas, E., Zelviene, P., Daniunaite, I., Hyland, P., Kvedaraite, M., Shevlin, M. et al. (2020). The structure of ICD-11 PTSD and complex PTSD in adolescents exposed to potentially traumatic experiences. Journal of Affective Disorders, 265, 169-174. https://doi.org/10.1016/j.jad.2020.01.061

Lechner-Meichsner, F. \& Steil, R. (2021). A clinician rating to diagnose CPTSD according to ICD-11 and to evaluate CPTSD sym- ptom severity: Complex PTSD Item Set additional to the CAPS (COPISAC). European Journal of Psychotraumatology, 12, 1891726. https://doi.org/10.1080/20008198.2021.1891726

Maercker, A., Brewin, C. R., Bryant, R. A., Cloitre, M., van Ommeren, M., Jones, L. M. et al. (2013). Diagnosis and classification of disorders specifically associated with stress. Proposals for ICD11. World Psychiatry, 12, 198-206. https://doi.org/10.1002/ wps. 20057

Matulis, S., Resick, P. A., Rosner, R. \& Steil, R. (2014). Developmentally adapted cognitive processing therapy for adolescents suffering from posttraumatic stress disorder after childhood sexual or physical abuse: A pilot study. Clinical Child and Family Psychology Review, 17, 173-190. https://doi.org/10.1007/ s10567-013-0156-9

McLaughlin, K. A., Koenen, K. C., Hill, E. D., Petukhova, M., Sampson, N. A., Zaslavsky, A. M. et al. (2013). Trauma exposure and posttraumatic stress disorder in a national sample of adolescents. Journal of the American Academy of Child \& Adolescent Psychiatry, 52, 815-813. https://doi.org/10.1016/j.jaac.2013. 05.011

Morina, N., Koerssen, R. \& Pollet, T. V. (2016). Interventions for children and adolescents with posttraumatic stress disorder. A meta-analysis of comparative outcome studies. Clinical Psychology Review, 47, 41 -54. https://doi.org/10.1016/j.cpr.2016. 05.006

National Institute for Clinical Excellence (NICE). (2018). Posttraumatic stress disorder (update). Guideline consultation. London, UK: NICE.

Perkonigg, A., Höfler, M., Cloitre, M., Wittchen, H.-U., Trautmann, S. \& Maercker, A. (2016). Evidence for two different ICD-11 posttraumatic stress disorders in a community sample of adolescents and young adults. European Archives of Psychiatry and Clinical Neuroscience, 266, 317-328. https://doi.org/10.1007/ s00406-015-0639-4

Reed, G. M. (2010). Toward ICD-11. Improving the clinical utility of WHO's International Classification of mental disorders. Professional Psychology: Research and Practice, 41, 457-464. https://doi.org/10.1037/a0021701

Roth, S., Newman, E., Pelcovitz, D., van der Kolk, B. \& Mandel, F. S. (1997). Complex PTSD in victims exposed to sexual and physical abuse: Results from the DSM-IV field trial for posttraumatic stress disorder. Journal of Traumatic Stress, 10, 539-555. https://doi.org/10.1023/A:1024837617768

Sachser, C. \& Goldbeck, L. (2016). Consequences of the diagnostic criteria proposed for the ICD-11 on the revalence of PTSD in children and adolescents. Journal of Traumatic Stress, 29, 120 - 123. https://doi.org/10.1002/jts.22080

Sachser, C., Keller, F. \& Goldbeck, L. (2017). Complex PTSD as proposed for ICD-11. Validation of a new disorder in children and adolescents and their response to trauma-focused cognitive behavioral therapy. Journal of Child Psychology and Psychiatry, 58, 160 -168. https://doi.org/10.1111/jcpp.12640

Schäfer, I., Borowski, J. \& Cloitre, M. (2019a) Behandlung der komplexen PTBS mit STAIR/Narrative Therapie. In Maercker A. (Hrsg.), Traumafolgestörungen (S. 311-330). Berlin: Springer. https://doi.org/10.1007/978-3-662-58470-5:16

Schäfer, I., Gast, U., Hofmann, A., Knaevelsrud, C., Lampe, A., Liebermann, P. et al. (2019b). S3-Leitlinie Posttraumatische Belastungsstörung. Berlin: Springer.

Steil, R., Fischer, A. \& Rosner, R. (2021). Internationale und deutsche Leitlinien zur Behandlung der PTBS bei Kindern und Jugendlichen. Ein Überblick über aktuelle Empfehlungen. Kindheit und Entwicklung, 30, 154-163.

Steil, R. \& Füchsel, G. (2006). Interviews zu Belastungsstörungen bei Kindern und Jugendlichen (IBS-KJ). Diagnostik der Akuten und der Posttraumatischen Belastungsstörung. Göttingen: Hogrefe. 
Steil, R. \& Rosner, R. (2009). Posttraumatische Belastungsstörung. Göttingen: Hogrefe.

Vasileva, M., Haag, A.-C., Landolt, M. A. \& Petermann, F. (2018). Posttraumatic stress disorder in very young children. Diagnostic agreement between ICD-11 and DSM-5. Journal of Traumatic Stress, 31, 529-539. https://doi.org/10.1002/jts.22314

Villalta, L., Khadr, S., Chua, K.-C., Kramer, T., Clarke, V., Viner, M. V. et al. (2020). Complex post-traumatic stress symptoms in female adolescents. The role of emotion dysregulation in impairment and trauma exposure after an acute sexual assault. European Journal of Psychotraumatology, 11, 1710400. https:// doi.org/10.1080/20008198.2019.1710400

World Health Organization (WHO). (1992). The ICD-10 classification of mental and behavioral disorders. Clinical descriptions and diagnostic guidelines. Geneva: WHO.
World Health Organization (WHO). (2018). ICD-11 Mortality and Morbidity Statistics. Geneva: WHO. https://icd.who.int/brow se11/l-m/en

\section{Förderung}

Open Access-Veröffentlichung ermöglicht durch die Universitätsbibliothek der Katholischen Universität Eichstätt-Ingolstadt.

\section{Rebekka Eilers, M.Sc. Psych.}

Katholische Universität Eichstätt-Ingolstadt

Fachbereich Psychologie

Ostenstraße 25

85072 Eichstätt

rebekka.eilers@ku.de 